\title{
DISTRIBUTION MAP OF PLANT FLUORESCENCE SPECTRUM IN THREE-DIMENSIONS CREATED BY A LASER-INDUCED FLUORESCENCE SPECTRUM (LIFS) LIDAR OBSERVATIONS
}

\author{
S. Utsunomiya, Y Saito*, Y. Kumagai, and T.Tomida.
}

Institute of Engineering, Shinshu University, 4-17-1 Wakasato, Nagano City, Nagano, 380-8553, Japan

*Email:saitoh@cs.shinshu-u.ac.jp

\begin{abstract}
We have developed a vegetation monitoring lidar called a laser-induced fluorescence spectrum (LIFS) lidar which is able to get the living status of plants by observing their fluorescence remotely. The features of its operation are; daytime observations possible even outdoors, mobility and self-sufficiency, capability of mapping plant living information, and a user-friendly operation by unifying the controls of different equipment of the lidar using software. These features make observations by our LIFS lidar possible at any time and any place. In forest observations, we could depict three-dimensional structures of fluorescence spectrums. We also discuss in this work the possibility of monitoring other plant physiological information such as the concentration of chlorophyll and photosynthesis secondary metabolites in this work.
\end{abstract}

\section{INTRODUCTION}

Photosynthesis, the most well-known activity of plants, supplies food and oxygen that is indispensable to our survival. Furthermore, various effects of the biological, chemical, and physical processes of plants influence the formation of the earth's natural environmental system. For example, gaseous biogenic volatile organic compounds (BVOCs) like aromatic compounds generate new compounds or exclude some through chemical reaction with various kinds of molecules in the Earth. ${ }^{[1]}$ Physical reactions in a forest such as the evaporation of a water droplet splashing on a leaf surface ${ }^{[2]}$ or a water micro-burst on the ground surface ${ }^{[3]}$ could be a great source of forest aerosol. These activities occur continuously, so information about the living status of a plant itself or vegetation such as forests or mountainsides is considerably important in maintaining the continuity of not only the earth's environment, but also human beings' society.
In this paper, we discuss the potential of the LIFS lidar, which we developed for such purposes, based on the results of field investigations.

\section{METHODOLOGY}

\subsection{Fluorescence spectroscopy}

Plants as living organisms need to be monitored using non-destructive techniques. Chemical analyses which involve picking, sampling, crushing plant parts or applying chemical liquid treatments should be avoided.

On the other hand, in plant science, many optical methods have been applied to understand the living status of plants. In particular, fluorescence spectroscopy is widely used and there are many reports on plant physiological monitoring using the fluorescence method for studying chlorophyll photosynthesis $^{[4]}$, photochemical reactions ${ }^{[5]}$, environmental-stress ${ }^{[6]}$ and others. In this work, we study the changes of fluorescence spectrum shapes of plants to gather such physiological information.

\subsection{Lidar remote sensing techniques}

Monitoring the living status of plants should be done under natural conditions where the plants grow, but these locations/places are often out of reach for humans. Lidar remote sensing techniques provide important possibilities in such situations.

\subsection{Laser-induced fluorescence spectrum (LIFS) lidar}

A fluorescence lidar that combines fluorescence spectroscopy and lidar remote sensing techniques can be a powerful apparatus in tree/forest surveys. We named this type of fluorescence lidar as a laser-induced fluorescence spectrum (LIFS) lidar and we can use it to investigate the living status of plants by monitoring the changes of their fluorescence spectrum shapes remotely. 


\section{CONSTRUCTION OF LIFS LIDAR SYSTEM}

\subsection{Requirements}

In the development of the LIFS lidar, we required the following functions.

1) Daytime and outdoor operation: As described above, photosynthesis is one of the key characteristics of plants and we require the LIFS lidar to be able to operate in daylight. This requires a technique for reducing the background sun which is a major source of noise in lidar observations.

2) Mapping: To survey the forest living status in which many plants and trees are mixed and grow at different locations, the system should have a function to create a three-dimensional structure of the distribution.

3) User-friendly operation: Because the system consists of several devices and equipment having different types of control mechanisms, it is necessary to unite the differences.

\subsection{Construction and performance check}

The configuration and specification of the LIFS lidar we developed based on the requirements in 3.1 are shown in Fig. 1 and in Table 1.

1) To enable daytime and outdoor operations, we adapted the synchronous detection technique ${ }^{[7]}$ and prepared a photonic multi-channel analyzer system (PMA-12 (C10029-01), Hamamatsu Photonics, Japan) whose detector was a gated intensified CCD. The CCD gate used as a lidar detector opens to coincide only with the arrival of fluorescence emissions corresponding to laser irradiations.

The actual effect of the synchronous detection technique on reducing the solar background was checked. In Fig. 2, we can see that the background intensity constantly decreases in proportion to the CCD gate time-width. The noise that was still left at $10 \mathrm{~ns}$ gate time-width appears to be the noise of the detection system itself which includes the thermal noise of the CCD device and the electrical noise of the system.

The size of the LIFS lidar was $2.5 \mathrm{~m} \times 0.5 \mathrm{~m} \times 0.8$ $\mathrm{m}$ and it operates on a stretcher using a small dynamo with power of $900 \mathrm{VI}$, so that it can be loaded in a small commercial van-type car which

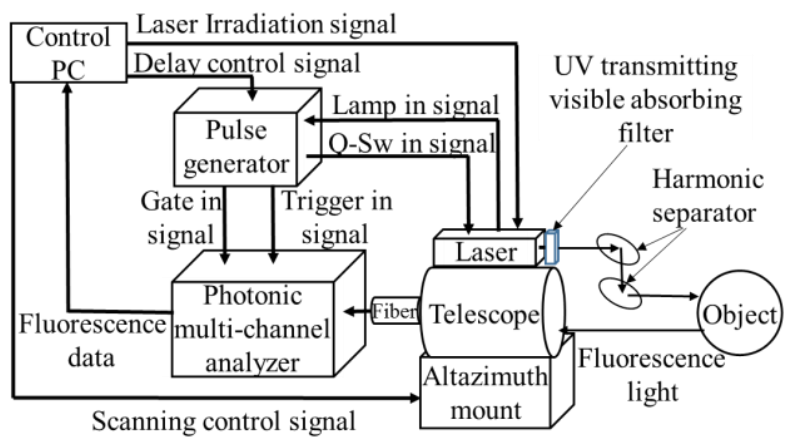

Fig. 1 Block diagram of the LIFS lidar. Operational parameter of each device and the flow of signals and data are shown.

Table I Specifications of the LIFS lidar

\begin{tabular}{|c|c|c|}
\hline Transmitter & & \\
\hline \multirow[t]{5}{*}{ Laser } & Wavelength & $355 \mathrm{~nm}$ \\
\hline & Pulse energy & $20 \mathrm{~mJ}$ \\
\hline & Pulse width & $6 \mathrm{~ns}$ \\
\hline & Reception & $10 \mathrm{~Hz}$ \\
\hline & Filter & Short pass $<400 \mathrm{~nm}$ \\
\hline \multicolumn{3}{|l|}{ Receiver } \\
\hline \multirow[t]{4}{*}{ Telescope } & Type & Schmidt- \\
\hline & & Cassegrainian \\
\hline & Effective area & $425 \mathrm{~cm}^{2}$ \\
\hline & Focal length & $2000 \mathrm{~mm}$ \\
\hline \multirow[t]{3}{*}{ Fiber } & Inlet & $1 \mathrm{~mm}$ \\
\hline & Outlet & Rectangle-shape \\
\hline & Filter & Long-pass $>370 \mathrm{~nm}$ \\
\hline \multicolumn{3}{|l|}{ Detector } \\
\hline \multirow[t]{2}{*}{ Spectrometer } & Range & 200-860nm \\
\hline & Resolution & $<3 \mathrm{~nm}$ \\
\hline \multirow[t]{2}{*}{ Sensor } & CCD & $\begin{array}{c}\text { Electronically cooled } \\
\text { type }\end{array}$ \\
\hline & Image Intensifier & 10ns gate \\
\hline \multicolumn{3}{|l|}{ Control/Processing } \\
\hline Delay/pulse generator & Resolution & $5 \mathrm{~ns}$ \\
\hline $\mathrm{PC}$ & OS & Windows 10 \\
\hline
\end{tabular}

is indispensable for outdoor observations such as forest investigations where the actual roadways to approach the place may be narrow and winding. This mobile and self-sufficient LIFS lidar arrangement made observations at any time and any place possible.

2) Mapping: A scanning function was added to the telescope operation. A laser beam is passed into the scanning area to get information at various depth. Horizontal and vertical scanning offers two-dimensional data at various distances and a three-dimensional structure of plant fluorescence can be made. 


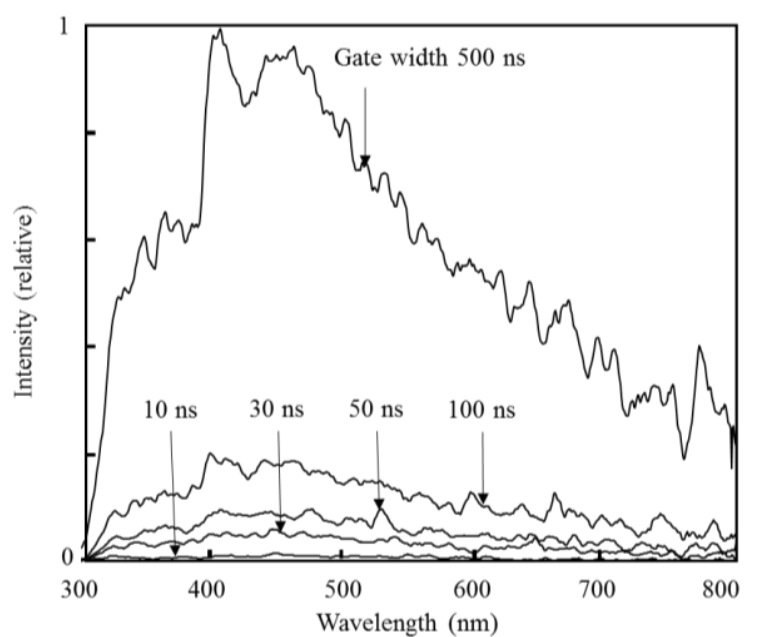

Fig. 2 Dependency of solar spectrum intensity on gate time-width.

3) User-friendly operation: We developed new software that could unite the operations of different devices/equipment. Each device had its own specifications for communication methods, software language, and operating systems. The flow of control of signals and data is shown in Fig. 1 and the structure of the new software for the LIFS lidar is shown in Fig. 3(a). The PMA was controlled only by GUI software with the AutoIt language that was provided by the manufacturer. Other pieces of equipment were unified in $\mathrm{C}++$ for maintainability. To be able to use these languages on a Microsoft Windows PC, a cygwin environment that allows programs of Unix-like systems to run was prepared. Each program was again integrated with the Ruby language and We were able to operate the LIFS lidar operation on the command line.

\section{OBSERVATIONS}

\subsection{Three-dimensional structures of chloro- phyll concentrations of a single tree}

Before conducting field observations, we checked the performance of the LIFS lidar. The lidar was positioned on a third floor veranda to point to the upper part of a zelkova tree.

To capture information at different depths, the distance to the object was changed by varying the delay-time of the CCD gate-open time in $10 \mathrm{~ns}$ increments which corresponds to a range resolution changes of $1.5 \mathrm{~m}$. The lidar scanned over an area of $13 \mathrm{~m}$ (horizontal distance, $\mathrm{H}$ ) with

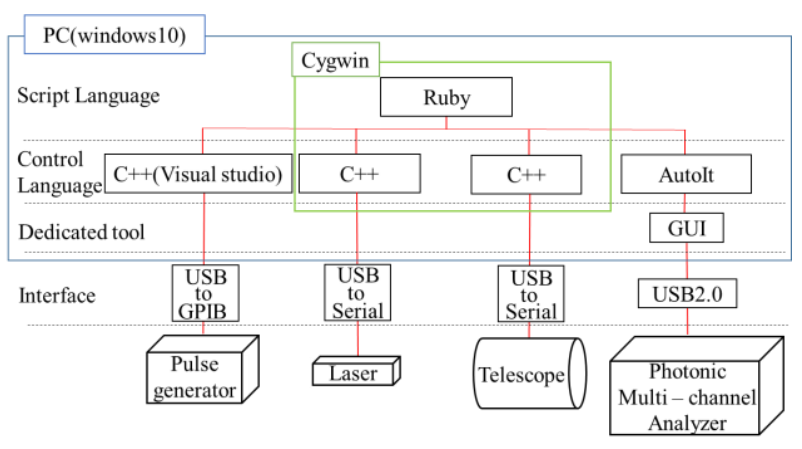

(a)

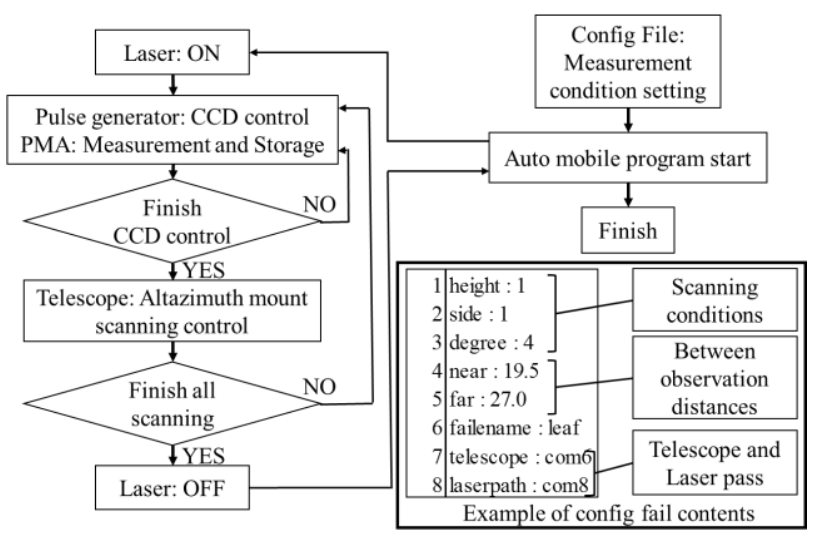

(b)

Fig. 3 Structure of the LIFS lidar software (a) and flow chart (b).

a horizontal angle resolution of 1 degree $\mathrm{x} 13 \mathrm{~m}$ (vertical distance, $\mathrm{V}$ ) with a vertical angle resolution of 1 degree.

It is known that the intensity ratio of chlorophyll fluorescence at $685 \mathrm{~nm}$ and $740 \mathrm{~nm}$ is an index of chlorophyll concentration ${ }^{[8]}$. The relationship between the ratio and chlorophyll concentrations measured by a high performance liquid chromatograph (HPLC) was investigated. The relationship was applied to the lidar observation result which offered the ratio. The distribution maps of chlorophyll concentrations of the zelkova tree are depicted in Fig. 4.

\subsection{Large scale structures of forest fluorescence spectrums}

Forest observations were done in locations where different kinds of trees grew naturally. Fig.5 shows the structure of fluorescence spectrum of vegetation. The covered area was $30 \mathrm{~m}(\mathrm{H})$ with a horizontal angle resolution of 3 degrees $\times 30 \mathrm{~m}$ (V) with a vertical resolution of 3 degrees $\times 20 \mathrm{~m}$ 


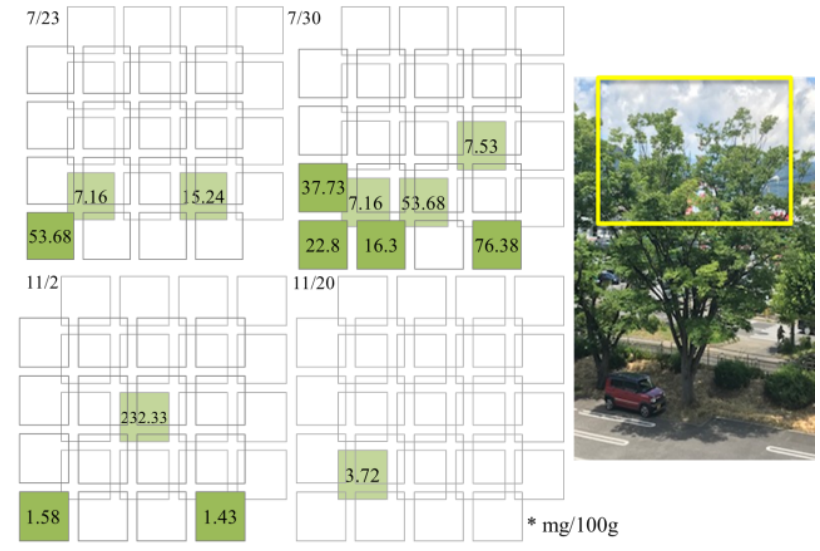

Fig. 4 Distribution map of chlorophyll concentration of a single zelkova tree.

(depth) with depth range resolution of $1.5 \mathrm{~m}$. The total number of pixels of the structure was 192. The range resolution in the depth direction was constant, but those in the horizontal and vertical directions became larger as the laser moved away from the system as shown in the figure.

The spectral shape appears to change depending on the tree location and type. Several papers report that plant fluorescence spectrums at around 400-650 nm involve information about photosynthetic secondary metabolites which are one of the key molecules to understand the status of plant growth. ${ }^{[5]}$ A detailed analysis of the lidar data still remains, but the usefulness of the LIFS lidar and the time effectiveness of constructing distribution maps in three-dimension was fully shown.

\section{CONCLUSIONS}

A mobile, self-efficient, automated, scanning LIFS lidar was developed and its performance was tested in the construction of threedimensional structures of plant fluorescence in daytime observations.

The distribution maps of chlorophyll concentrations of a single tree clearly showed differences in leaf location and in season. In forest observations, the fluorescence spectrum of different kinds of trees distributed over a large area could be obtained.

The growth status of vegetation may complexly depend on environmental factors such as soil, water, air, and others. We have already checked that our LIFS lidar has the potential to detect fluo-

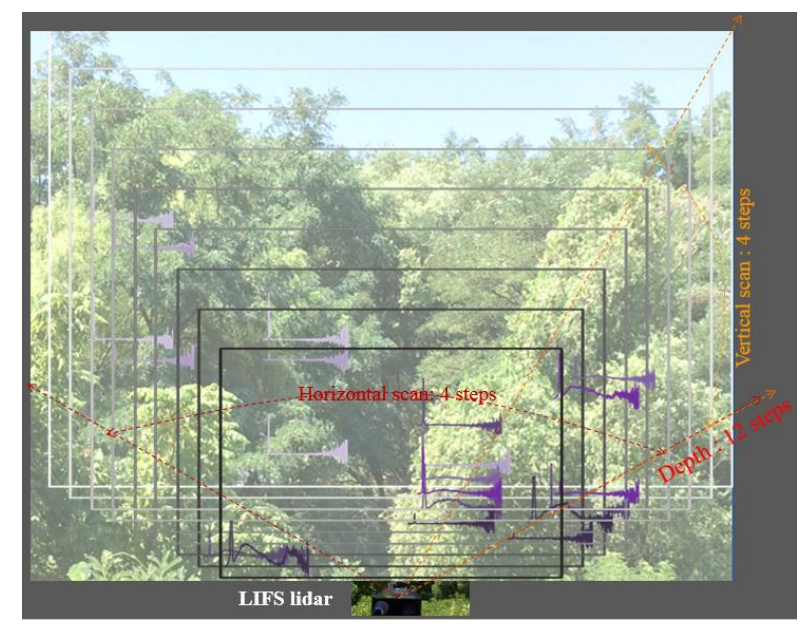

Fig. 5 Distribution map of the fluorescence spectrum of a forest.

rescence for such parameters ${ }^{[9]}$ Our interest is a fusion experiment of monitoring plant living information together with plant-growth environment conditions using the LIFS lidar.

\section{ACKNOWLEDGEMENTS}

This work was supported by the $27^{\text {th }}$ Botanical Research Grant of The New Technology Development Foundation and the joint research program of CEReS at Chiba University.

\section{REFERENCES}

[1] Y. Saito et al., Applied Optics, Vol. 40, pp. 3572-3574, 2001.

[2] S. Murakami, J. Hydrol., Vol. 319, pp.72-82, 2006.

[3] Y. S. Joung and C. R. Buie, Nat. Commun, Vol. 6, 6083, 2015.

[4] Chlorophyll a Fluorescence (G. C. Papageorgiou and Govindjee Eds.), Springer, 2004.

[5] Z. G. Cerovic et al., Agronomie 19, pp.543578, 1999.

[6] Y. Saito, in Recent Progress of Bio/Chemiluminescence an Fluorescence Analysis in Photosynthesis (N. Wada an M. Mimura Eds.), Chapter 11, Research Signpost, 2005.

[7] Y. Saito et al., Applied Optics, Vol.\#55, pp.6727-6734, 2016.

[8] H. K. Lichtenthaler, J. Plant Physiol. 131, pp.101-110, 1987.

[9] Y. Saito et al., Proceeding of SPIE, Vol. 10779 107790M; doi: 10.1117/12.2324428. 